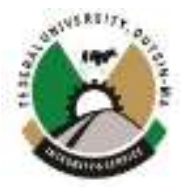

\title{
DETERMINING THE OPTIMUM MATURITY OF MAIZE USING GOOGLENET
}

\author{
Peter, A. \\ Department of Mathematical Sciences, Kaduna State University, Kaduna, Nigeria \\ Corresponding Author's Email: ayubng@kasu.edu.ng
}

\begin{abstract}
Climatic changes, animal and human activities that lead to desertification and deforestation have affected the increase in agricultural produce especially in sub-Sahara Africa. Several efforts have been put in place to reduce these effects. However, that has not fully resolved the problem food shortages due to the growing population in sub-Sahara Africa. The application of image processing and convolutional neural network in the determination of the optimum maturity of SAMMAZ 17 variety of maize plant is being considered to mitigate for the shortage of food production. The optimum maturity is determined by using GoogleNet pre trained network on 3000 samples of maize comb captured using a camera at different maturity stages in a farmland. GoogleNet pre-trained network gave an accuracy of $82.44 \%$. The result obtained showed a $10.44 \%$ improvement over an earlier result using Alexnet pre-trained network. The results suggest that when made operational there is a window of opportunity for increase in the production of food in sub-Sahara Africa.
\end{abstract}

Keywords: Convolutional Neural Networks, Maize, Optimum Maturity, Googlenet

\section{INTRODUCTION}

There is several challenges cause by changes in climatic conditions in sub-Sahara Africa. In Nigeria, being the most populous African country is faced with these challenges and those cause by human activities. Notable amongst these problems are deforestation and desertification. These have led to shortages in food supply as a result of inadequate land for grazing and agricultural activities, conflicts, loss of lives and properties (Peter, Abdulkadir, \& Abdulhamid, 2017; Gambari, 2018). The increase in both human and livestock has necessitated the generation of different varieties of crops to combat desert encroachment in both lowlands and uplands in sub-Sahara Africa. This is to ensure the supply of adequate food for sustenance. The maize plant is one amongst the many agricultural crops that can be cultivated under different climatic conditions and meets the nutritional requirement of both humans and livestock in sub-Sahara Africa (Olaniyan, 2015; Peter, Damuut \& Abdulkadir, 2020). The common method of harvesting maize in sub-Sahara Africa is at its biological maturity. At this stage, the nutrient level has seriously decline, and livestock do not usually benefit much when grazing on the maize plant stalk; sometimes the stalk are burnt. The maize maturity periods in term of days varies from one variety to the other. However, their maturities in terms of visual features like changes in coloration, texture, etc. are common amongst several varieties of maize. The use of vision to determine maize maturity is not very reliable as human eyes have differences in vision, weariness of sight, fatigue, etc., these can impair the right judgment. The use of image processing and Neural Network $(\mathrm{NN})$ in determining the optimum maturity of maize for harvesting has mitigated for the classical approach of using human eyes (Peter, Damuut \& Abdulkadir, 2020).

Convolutional Neural Networks (CNNs), or ConvNet are unique multilayer NNs that detect visual configurations from picture elements (pixel) image from little preprocessing of the images acquired. In this section, literature review of related works on the identification and classification of agricultural produce using CNNs techniques are presented. CNN was applied in Yulcin \&Razavi (2016) for detecting sixteen varieties of beans, cherries, pomegranates, and apricot, the classification result is 97.47\% accuracy. Suma, Shetty,Tated, Rohan \& Pujar (2019) used CNN to identify and classify the images of leaves from a common location, and obtained a classification accuracy of $99.32 \%$. Prashar \& Kant (2019) in an effort to reduce loss in profit by cotton farmers in India reported the use of CNN model having overlapping pooling technique and many layer perceptron methods in the identification and classification of good leaves. 96\% identification accuracy was obtained. Boulent, Foucher, Theau, \& St-Charles (2019) used 3 set of images under different conditions and applied CNN in the classification of crop diseases. They claimed that $\mathrm{CNN}$ gives better performances when dealing with complex situations. Jiang, Chen, Liu, He, \& Liang (2019) improved upon the extraction potential of CNN by introducing a Nobel identification method from Single-Short Mult-box Detector (SSD) with GoogleNet Inception unit. $78.80 \%$ identification accuracy was obtained when the innovation was applied on five infectious leaf diseases. Lin et al. (2019) proposed a matrix-based CNN (M-bCNN) and used it in classifying eight categories images that were augmented, and obtained $96.5 \%$ validation accuracy and $90.1 \%$ testing accuracy. They claimed their results are better than those generated by pretrained Alexnet and VGG-16. Zeiler and Fergus networks were modified in the work of Li, Chen, Wang, \& Xie (2019), the resulting CNN reported a classification accuracy of $88.5 \%$ when applied in the identification of wheat mite. Suresh, Gnanaprakash, \& Santhiya (2019) reported that Inception 
version 3 gave better results than ResNet50 version2 and MobileNet version2 when applied in the identification and classification of three types of infections on grape fruit. Ghostly features of Com seeds were extracted using CNN and cold damages was estimated in (Yang, Yang, Hao, Xie, \& Li, 2019). $\mathrm{CNN}$ has a great potential for improving identification and classification accuracies in plant diseases (Saleem, Potgieter, \& Arif, 2019). Gonzalez, Arellano, \&Tapia (2019) compared ResNet50, ResNet101, and Mobile-Net version1 in the identification of blueberry images; RestNet50 gave a better result with $75.90 \%$ mean precision. Faster R-CNN combination and K-Means Clustering method was compared in the identification of certain rice diseases, and the results showed a very impressive performance (Zhou, Zhou, Chen, \& He, 2019). In the work reported in Elhassouny \& Smarandache (2019) shows that lower learning rates in identification and classification of Tomatoes leaf diseases generates better results. $\mathrm{Li}$ et al. (2019) combined CNN methods and density approximation in counting soya beans which is promising in breeding task. Combined approaches of Gated Recurrent Unit Networks and CNNs in differentiating similar crops have yielded excellent results (Li, Chen, \& Zhang, 2019). Average classification accuracy of $99.58 \%$ was obtained in predicting maize production using CNN model (Abdullahi, Sheriff, \& Mahieddine, 2017). Li, Xia, Du, Lin, \& Samat (2017) altered the number of pooling layers and convolutional layers in three pretrained networks to improve their performance. The results obtained suggest a correlation between the accuracy of classification and the number of layers as seen in (Sinha, Verma, \& Haidar, 2017; Haryanto, Wasito, \& Suhartanto, 2017). Zhang et al. (2018) obtained $91.90 \%$ accuracy within a time less than 0.01 predictions by using $\mathrm{CNN}$ in determining the accuracy and scalability of Tomatoes. A general network model based on Unet architecture was used to classify and segment several organs in the medical field. The results are $99 \%$ for classification and for segmentation $89 \%$ (Harouni, Karargyris, Negahdar, Beymer, \& Syeda-Mahmood, 2018). Xia et al. (2018) used ANN and Support Vector Machine (SVM) in the identification and classification of matured varieties of oilseeds. ANN outperformed SVM in their results. $\mathrm{Hu}$ et al. (2016) stated that though Time series can explain seasonal characteristics of agricultural crops, they are not good in classification tasks. Do,
Kim, Yang, Lee, \& Na (2018) reported facial identification and non-facial identification and classification task by $\mathrm{CNN}$.

The use of ANN methods are reported in (Peter et al., 2017; Peter \& Abdulkadir, 2018) for determining the optimum ripeness of maize from its leaves. However, Peter et al. (2020) stated that maize leaves are not very reliable for classification purposes. And the maize variety is of importance as their maturity period varies as well as climatic conditions for cultivation as reported in (Garba \& Namo, 2013). Maize comb can give beneficial outcome to herders and crop farmers (Konar, 2005). NNs possess the capability of distinguishing patterns (Bezdek, 1994). In the work reported in Peter et al. (2020), Resnet50 outperformed the orthodox ANN. However, the performance of Resnet50 in the classification of the maize comb is not quite impressive. The non-refinement of layers must have affected the performance of Restnet50. The need to refine the layers or perhaps use a different pre-trained CNN to enhance the result in order to obtain a better classification when made operational is inevitable. In this paper, GoogleNet, a pioneer and deep Convolutional Neural Network (CNN) that utilizes the Inception module leveraging on feature identification at diverse measures through convolution with diverse filters with the result of dimensionality reduction by means of $1 \mathrm{x} 1$ convolution block which reduces the arithmetic operations in the layers. This characteristic has made Googlenet resolve the problems of over fitting, fading gradient or explosion that arises when there are many convolutional layers and units in a network, though with increase performance. (Zhang, Allen, Unger, \& Cruz, 2018). The current work is an extension and enhancement upon the work in (Peter et al., 2020) which has not been address in this way before. The paper is organized as follows: Section 2 gives the approach used in cultivating the maize variety, image acquisition and classification. The results and discussions are presented in Section 3. Section 4 concludes the paper with future research direction and recommendations.

\section{MATERIALS AND METHODS}

SAMMAZ 17 maize variety, acquired maize comb images and the capturing device represent the materials. The method of processing images and classifying them represents the methods. The system framework is presented in Figure 1. 
Resizing of images to fit the input layer "conv" dimension [224 224 3] of GoogleNet

\begin{tabular}{|l|}
\hline $\begin{array}{l}\text { Loading of the folders containing the resize } \\
\text { images to MATLAB work space }\end{array}$ \\
\begin{tabular}{|l|} 
Creating an image Data Store that operates on \\
each folder as a category
\end{tabular} \\
\hline $\begin{array}{l}\text { Counting and randomly picking } 1000 \text { images } \\
\text { in each folder as a category }\end{array}$ \\
\hline $\begin{array}{l}\text { Images are augmented to reduce over fitting } \\
\text { problem during training }\end{array}$ \\
\hline $\begin{array}{l}\text { Images are randomly each divided into } 70 \% \text { for } \\
\text { training and the rest for testing }\end{array}$ \\
\hline
\end{tabular}

Figure 1: Framework of the System

\section{The Maize Variety}

The maize variety was SAMMAZ 17; a potent variety of maize suitable for different climatic conditions was cultivated for the research. It has a high resistance for drought, striga conditions with high nutrients (Garba \& Namo, 2013; Garba, Ahmed, Katung, Lawal, \& Abubakar, 2017).

\section{Climatic Condition}

SAMMAZ 17 maize variety was cultivated in highland. The area has an average daily temperature of $83^{\circ} \mathrm{F}$ from the months of June to October with a probable rainfall of 0.46 inches as reported in (Gelaro et al., 2017).

\section{Selection of Farming Site and Preparation}

A three hectare farm site having good drainage was used for the cultivating of SAMMAZ 17 maize variety. In an effort to guarantee that glyphosate did not affect the maize seeds while destroying the weeds, the farm site was left for two weeks after glyphosate application before plowing, harrowing and ridging.

\section{Pre-emergence}

Two and half liters of Paraquat and five sachets of combi plus are prepared in one litre of water per hectare and used to destroy weed seeds disperse after application of glyphosate.

\section{Post-emergence}

After the germination of the maize, two liters herbicide (guard force) and powder sachets were applied per hectare. Three bags of NPK were applied after 2 weeks. Additional three bags of
NPK were applied after 5 weeks of plantation. Two bags of Urea fertilizer were applied per hectare during the eighth week.

\section{Image Collection}

A thousand maize comb images at the $10^{\text {th }}$ week of cultivation were captured using a Full High Definition Charge Couple Device (CCD) digital camera FINEPIX Z35 placed at $90^{\circ}$ and 3 inches from the maize comb. The capturing of images was repeated at the $11^{\text {th }}$ and $12^{\text {th }}$ week. The differences in the period of image acquisition represents the maturity periods which are soft dough stage, hard dough stage and optimum maturity stage respectively.

\section{Experimental setup}

MATLAB R2018a software running on Windows7 Ultimate Operating System 64-bit service pack1 was used for the experiment. The processor is Intel ${ }^{\circledR}$ Core(TM) i7 with $2620 \mathrm{M}$ CPU@2.70GHz. 8.00GB RAM size and 500GB HDD.

Preparation of Images for GoogleNet pre-trained network The images were resized to fit the input layer "Conv" having an input size of [ [224 224 3], which stand for 224 rows by 224 columns with three channels of the RGB image format.

\section{Processing of Images}

The resized images were labeled asLowRGB224, MediumRGB224 and HighRGB224 in three different folders representing the soft dough stage, hard dough stage, and the maturity stage respectively. The images are loaded on the 
MATLAB work space and by applying imageDatastore () method an image data store (imds) was formed which assist in managing data by working on the image folder. The numbers of images in each category were randomly updated to one thousand each and used for input.

\section{RESULTS AND DISCUSSIONS}

\section{Image Training}

The input images from each category are randomly divided into $70 \%$ for training and the rest for testing. The initial conv layer reduced the input images through the use of large filters, however, the three-dimensional information were retained. In the second conv layer the image sizes are further reduced by four times and eight times prior to the first inception module, however, generating very high number of feature maps. Also at the second conv layer the computational burden are drastically reduced through the $1 \times 1$ conv block. The max pool layers located in between the inception modules primarily reduce the height and width of the input data when fed forward in the network. This equally reduces the computational burden. The average pooling layer calculates the mean of all the feature maps generated in the second conv layer and subsequently decrease the image height and width to $1 \times 1$. In order to ensure that over fitting is reduced during training, the dropout layer is used to regularize the number of neurons interconnected within the network. To further regularize and reduce over fitting an auxiliary classifier is embedded within the third and sixth Inception modules during training. This is to guarantee that classification is performed based on the input. The components in the auxiliary classifier are average pool layer, conv layer, 2 fully connected layers, dropout layer and softmax layer with the softmax activation function. Finally, to ensure the desired event occurs for each class, the softmax activation function was used at the softmax layer being the final layer. The learning rate is 0.0001 , stochastic gradient descent with momentum, 20 epochs are used. Figure 2 and figure 3 are the results of the classification.
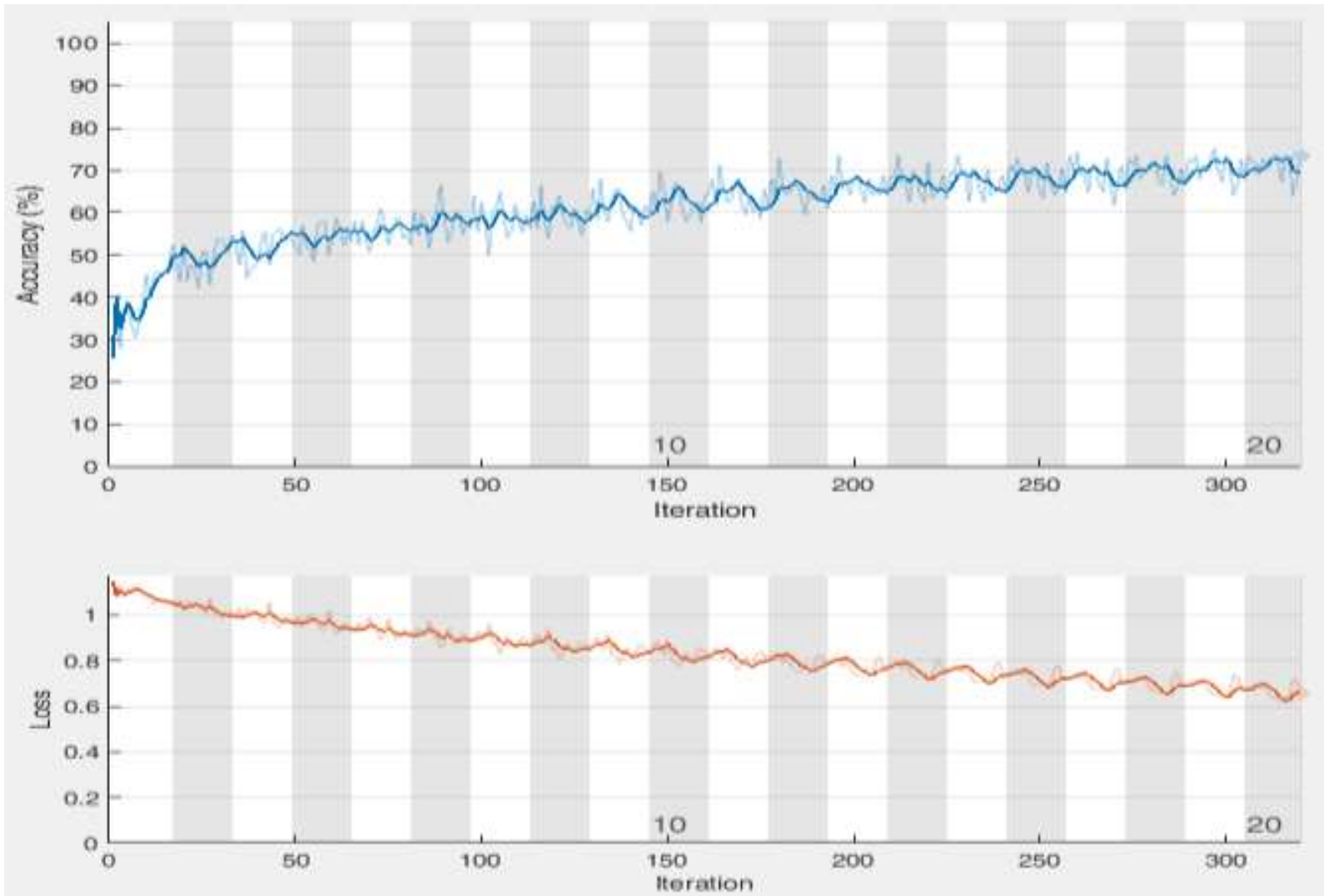

Figure 2: Classification Performance of GoogleNet 


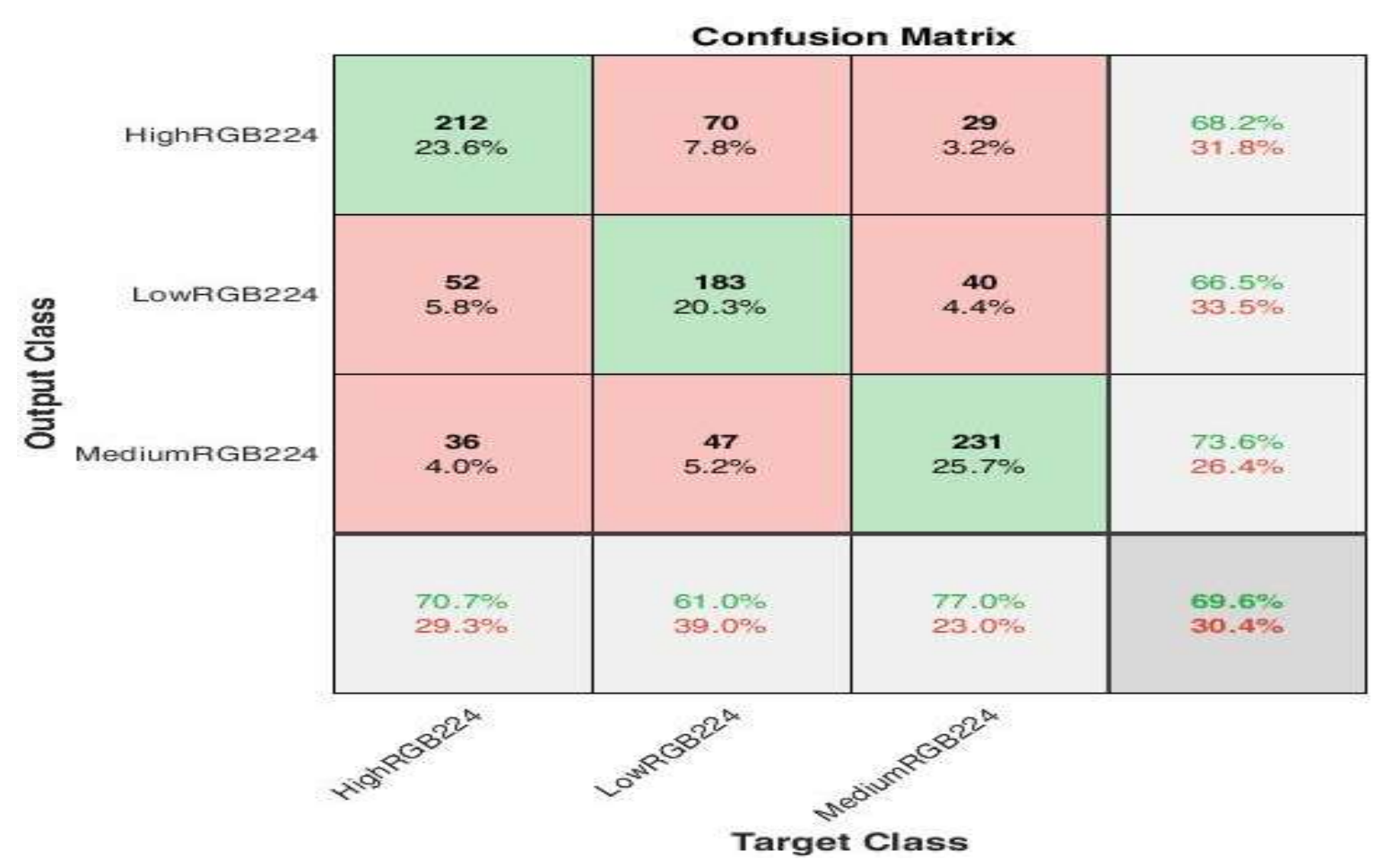

Figure 3: Confusion Matrix

In Figure 2, the accuracy of the GoogleNet classifier is about $70 \%$ and the loss is around 0.6 which is fairly above the result reported in (Peter et al., 2020). In Figure 3, being the confusion matrix depicts the target class verses the output class. The HighRGB224 category, being the optimum matured maize is correctly classified by $68.2 \%$ and wrongly classified as $31.8 \%$. In the MediumRGB224 category, being the hard dough stage is correctly classified as $73.6 \%$ and $26.4 \%$ misclassified. Finally, the LowRGB224 categories, being the soft dough stage, $66.5 \%$ are correctly classified and $33.5 \%$ are misclassified. The overall accuracy of classification is $69.6 \%$.

\section{CONCLUSION}

In this paper, an extension and improvement upon an earlier work reported in Peter et al. (2020) is presented. The work attempts to encourage the integration of farmers and herders by proposing an optimum period of harvesting maize crop that would allow for herders to graze on the fresh maize stalk. This would encourage more food production, mutual and peaceful coexistence between herders and farmers. The ANN variant used is the deep NN of the GoogleNet pre-trained network. An auxiliary classifier concept was applied to the third and sixth Inception module to ensure regularization and reduce over fitting problem prior to the classification. An average classification accuracy of $69.6 \%$ was obtained. The result shows that the network can identify the hard dough stage more followed by the optimum mature stage.

\section{Future Research Direction}

The findings suggest a window for future work in the area of classifying maize on the basis of optimum maturity in other to improve food production and betterment of farmers and herders livelihood.

\section{Data Availability}

Upon request the data of the maize comb images are available. Conflict of Interest

The author declares that there is no conflict of interest regarding the publication of this paper.

\section{Funding Statement}

This work was supported by Tertiary Education Trust Fund (TET Fund) of Nigeria under Institutional Based Research (IBR) Grant 2018 through Kaduna State University, Kaduna.

\section{Acknowledgements}

The author acknowledges the Almighty God for the inspiration to undertake the research. The author equally appreciates the efforts of Zachariah Babangida and Barna Thomas Lass in the Department of Computer Science, Kaduna State University for improving the result of this work. Mrs Peter Rahila Ayuba is also appreciated for her expertise in cultivating the maize and data acquisition.

\section{REFERENCES}

Abdullahi, H., Sheriff, R., \& Mahieddine, F. (2017). Convolution neural network in precision agriculture for plant image recognition and classification. 2017 Seventh International Conference on Innovation, Computing Technology (Intech.), IEEE, Londr'es, 1-3. 
Bezdek, J. C. (1994). What is Computational Intelligence? In Computational Intelligence Imitating Life, Zurada, J. M., Marks, R. J. and Robinson, C. J. (Eds), IEEE Press, NY, 1-2.

Boulet, J., Foucher, S. Theau, J., \& St-Charles, P-L. (2019). Convolutional neural networks for the automatic identification of plant diseases. Frontier in Plant Science, 10(941), 1-15.

Do, N., Kim, S., Yang, H., Lee, G. \& Na, I. (2018). Face Tracking with Convolutional Neural Network Heat Map. Proceeding of International Conference on Machine Learning and Soft Computing ICMLSC,2018) February 2-4, ACM, 41-44.

Elhassouny, A., \& Smarandache, F. (2019). Smart mobile application to recognize tomato leaf diseases using convolutional neural networks. IEEE/ICCSRE, 22-24 July, Agadir, Morocco, 2019, 1-4.

Gambari, I. A. (2018). Solution to Farmers' Herders Conflict," UNIUYO New Bulletin, 41(3), 4-6.

Garba, L.L., \& Namo, O.A.T. (2013). Productivity of maize hybrid maturity classes in savanna agro-ecologies in Nigeria. African Crop Science Journal, 21(4), 323-335.

Garba, Y., Ahmed, B., Katung, M. D., Lawal, A. F., \& Abubakar, H. N. (2017). Profitability of striga tolerant maize variety (SAMMAZ 17) amongst smallholder farmers in Lapai, Niger State, Nigeria. South African Journal of Agricultural. Extension, .45(1), 1-9.

Gelaro, R., McCarty, W., Suarez, M. J., Todling, R., Molod, A., Takacs, L., ..., Zhao, B. (2017). The modern-era retrospective analysis for research and applications, Version 2 (MERRA-2). Journal of Climate, 30, 5419-5454.

Gonzalez, S., Arellano, C., \& Tapia, J. E. (2019). Deep blueberry: Quantification of blueberries in the wild using instance segmentation. IEEE Access, Special Section on New Technology for Smart Farming 4.0: Research Challenges and Opportunities, Multidisciplinary Rapid Review Open Access Journal.,7, 105776-105788.

Harouni, A., Karargyris, A., Negahdar, M., Beymer, D., \& Syeda-Mahmood, T. (2018). Universal multi-modal deep network for classification and segmentation of medical images. 2018 IEEE $15^{\text {th }}$ International Symposium on Biomedical Imaging (ISBI 2018) April 4-7, Washington, D.C., USA, 872876.

Haryanto, T., Wasito, I., \& Suhartanto, H. (2017). Convolutional neural network (CNN) for gland images classification. Information \& Comm. Technol. and System (ICTS). 2017 11th International Conference on IEEE, 55-60.

Hu, Q., Wu, Q., Song, Q., Yu, Q., Lu, M., Yang, P., ..., Long, Y. (2016). Extending the pairwise separability index for multi-crop identification using time-series MODIS images. IEEE Transaction on Geoscience and Remote Sensing, 54(11), 6349. 6361.

Jiang, P., Chen, Y., Liu, B., He, D., \& Liang, C. (2019). Realtime detection of apple leaf diseases using deep learning approach based on improved convolutional neural networks. IEEE Access, Special Section on Advancement Optical Image for Extreme Environments. IEEE Access, Multidisciplinary Rapid Review Open Access Journal, 7, 59069-59080.

Konar, A. (2005). Computational Intelligence: Principles, Techniques and Applications, Springer Berlin Heidelberg New York, 4-5.

Li, E., Xia, J., Du, P., Lin, C., \& Samat, A. (2017). Integrating multilayer features of convolutional neural networks for remote sensing scene classification. IEEE Transaction on Geoscience and Remote Sensing, 55(10), 5653-5665.

Li, W., Chen, P., Wang, B. \& Xie, C. (2019). Automatic localization and count of agricultural crop pest based on an improved deep learning pipeline. Science. Report, 9(7024), 111.

Li, Y., Jia, J., Zhang, L., Khattak, A., Sun, S., Gao, W., \& Wang, M. (2019). Soybean seed counting based on pod image using two-column convolution neural network. IEEE Access, Multidisciplinary Rapid Review Open Access Journal, 7, 64177 64185.

Li, Z., Chen, G., \& Zhang, T. (2019). Temporal attention networks for multi-temporal multi-sensor crop classification. IEEE Access, Multidisciplinary Rapid Review Open Access Journal, 7, 134677-134690.

Lin, Z., Mu, S., Huang, F., Abdulmateen, K., Wang, M., Gao, W., \& Jia, J. (2019). A unified matrix-based convolutional neural network for fine-grained image classification of wheat leaf diseases. IEEE Access, Multidisciplinary Rapid Review Open Access Journal, 7, 11570-11590.

Muzhingi, T., Gadaga, T. H., Siwela, A. H., Grusak, M. A., Russell, R. M., \& Tang, G. (2011). Yellow maize with high betacarotene is an effective source of vitamin A in healthy Zimbabwean men. American. Journal of Clinical Nutrition., 94(2), 510-519.

Olaniyan, A. B. (2015). Maize: Panacea for Hunger in Nigeria. African. Journal. of Plant Science, 9(3), 155-174.

Peter, A. \& Abdulkadir, S. (2018). Application of image processing and neural networks in determining the readiness of maize. Proceedings of International Conference on Machine Learning and Soft Computing (ICMLSC 2018) February 2-4, 2 ACM, 104-108.

Peter, A. Abdulkadir, S. \& Abdulhamid, U. F. (2017). Detection of optimum maturity of maize using image processing and artificial neural networks. Science World Journal, 12(2), 24-27.

Peter, A. Damuut, L. P., \& Abdulkadir, S. (2020). Determining the optimum maturity of maize using computational intelligence techniques. American Journal of Neural Networks and Applications, 6(1), 1-9.

Pingali, P. L. (2011). Green Revolution: impacts, limits, and the path ahead. Proceedings of the National Academic of Science of the United States of America, 12302-12308.

Prashar, K., Talwar, R., \& Kant, C. (2019). CNN based overlapping pooling method and multi- layered learning with SVM \& KNN for American cotton leaf disease recognition. 
2019 International Conference on Automation, Computational and Technology Management (ICACTM) Amity University, IEEE, 330-333.

Saleem, M. H., Potgieter J., \& Arif, K. M. (2019). Plant disease detection and classification. Plants, 8(468), 1-24.

Sinha, T., Verma, B., \& Haidar, A. (2017). Optimization of convolutional neural network parameters for image classification. Computational Intelligence (SSCI). 2017 IEEE Symposium Series on IEEE, 1-7.

Suma, V., Shetty, R. A., Tated, R. F., Rohan, S. \& Pujar, T. S. (2019). CNN based leaf disease identification and remedy system. Proceedings of Third International Conference on Electronics Communication and Aerospace Technology (ICECA 2019). IEEE Conf. Record \#45616; IEEE Xplore ISBN: 978-17281-0167-5, 395-399.

Suresh, G., Gnanaprakash, V., \& Santhiya, R. (2019). Performance analysis of different cnn architecture with different optimizers for plant disease classification. 2019, $5^{\text {th }}$ International Conference on Advanced, Computing and Communication Systems (ICACCS), IEEE, 916-921.

Xia, J., Yang, Y., Cao, H., Zhang, W., Xu, L., Wan, Q., ..., Huang, B. (2018). Hyperspectral identification and classification of oilseed rape waterlogging stress levels using parallel computing. IEEE Access, Multidisciplinary Rapid
Review Open Access Journal, 6, 57663-57675.

Yang, W., Yang, C., Hao, Z., Xie, C., \& Li, M. (2019). Diagnosis of plant cold damage based on hyperspectral imaging and convolutional neural network. IEEE Access, Multidisciplinary Rapid Review Open Access Journal, 7, 118239-118248.

Yulcin, H. \& Razavi, S. (2016). Plant classification using convolutional neural networks, in Agro-Geoinformatics (Agro Geoinformatics), 2016 Fifth International Conference on IEEE, $1-5$.

Zhang, L., Jia, J., Gui, G., Gao, W., \& Wang, M. (2018). Deep learning based improved classification system for designing tomato harvesting robot. IEEE Access, Special Section on AIDriven Big Data Processing: Theory, Methodology and Applications. IEEE Access, Multidisplinary Rapid Review Open Access Journal, 6, 67940-67950.

Zhang, Y., Allen, J., Unger, J. B. \& Cruz, T. B. (2018). Automated identification of lookahs (water pipes) on Instagram: An application in feature extraction using convolutional neural network and support vector machine classification. Journal of Medical Internet Research, 20(11), 1-15.

Zhou, G., Zhuo, W., Chen, A., \& He, M. (2019). Rapid detection of rice disease based on FCM-KM and faster R-CNN fusion. IEEE Access, Multidisciplinary Rapid Review Open Access Journal, xx,1-19. 